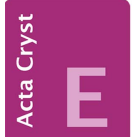
COMMUNICATIONS

ISSN 2056-9890

\section{Crystal structure of 3-bromoacetyl-6- chloro-2H-1-benzopyran-2-one}

\author{
Ramanaiah Chennuru, ${ }^{\text {a }}$ Balaji Maddimsetti, ${ }^{\mathrm{a}}$ Suman \\ Gundlapalli, ${ }^{\mathbf{b}}$ R. Ravi Chandra Babu ${ }^{\mathbf{b}}$ and Sudarshan \\ Mahapatra $^{\mathrm{a} *}$
}

${ }^{a}$ Dr Reddys Laboratory, Innovation Plaza, IPDO, Bachupally, Hyderabad 500090 India, and ${ }^{\mathbf{b}}$ GITAM University, Department of Chemistry, College of Science,

Vishakapatnam, Andhrapradesh, India. *Correspondence e-mail: sudiisc@gmail.com

Received 17 June 2015; accepted 6 July 2015

Edited by A. J. Lough, University of Toronto, Canada

In the title compound, $\mathrm{C}_{11} \mathrm{H}_{6} \mathrm{BrClO}_{3}$, the benzopyran ring system is essentially planar, with a maximum deviation of 0.036 (2) $\AA$ for the $\mathrm{O}$ atom. The $\mathrm{Cl}$ and $\mathrm{Br}$ atoms are displaced by -0.0526 (8) and 0.6698 (3) $\AA$, respectively, from the mean plane of this ring system. In the crystal, two pairs of weak $\mathrm{C}-$ $\mathrm{H}$... O hydrogen bonds to the same acceptor $\mathrm{O}$ atom link molecules into inversion dimers.

Keywords: crystal structure; coumarin; hydrogen bonding.

CCDC reference: 739322

\section{Related literature}

For applications of coumarins, see: Kale \& Patwardhan (2014); Eid et al. (1994); Hsieh (2015); Ballazhi et al. (2015); Wang (2015); Lanoot et al. (2002); Morris \& Russell (1971); Hooper et al. (1982); Khalfan et al. (1987). For related structures, see: Munshi et al. (2004); Munshi \& Guru Row (2006); Chopra et al. $(2006,2007 a, b)$.

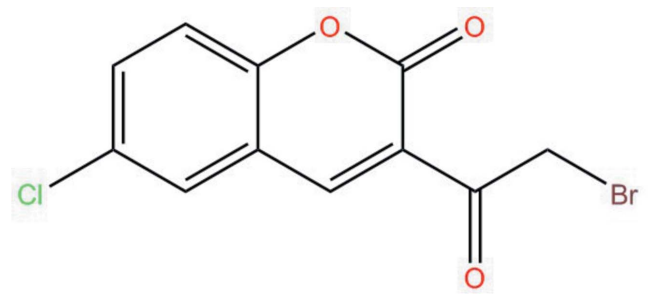

\section{Experimental}

\subsection{Crystal data}

$\mathrm{C}_{11} \mathrm{H}_{6} \mathrm{BrClO}_{3}$

$M_{r}=301.51$
Monoclinic, $P 2_{1} / c$

$a=12.5770(2) \AA$

$b=5.7977$ (1) $\AA$

$c=14.8390(3) \AA$

$\beta=94.679$ (2)

$V=1078.42(3) \AA^{3}$

\subsection{Data collection}

Bruker SMART CCD area-detector diffractometer

Absorption correction: multi-scan (SADABS; Sheldrick, 1996)

$T_{\min }=0.295, T_{\max }=0.712$

\subsection{Refinement}

$R\left[F^{2}>2 \sigma\left(F^{2}\right)\right]=0.030$

$w R\left(F^{2}\right)=0.076$

$S=0.95$

2113 reflections

145 parameters

$\mathrm{H}$-atom parameters constrained

$\Delta \rho_{\max }=0.43{\mathrm{e} \AA^{-3}}^{-3}$

$\Delta \rho_{\min }=-0.60 \mathrm{e}^{-3}$

Table 1

Hydrogen-bond geometry $\left(\AA{ }^{\circ}\right)$.

\begin{tabular}{lllll}
\hline$D-\mathrm{H} \cdots A$ & $D-\mathrm{H}$ & $\mathrm{H} \cdots A$ & $D \cdots A$ & $D-\mathrm{H} \cdots A$ \\
\hline $\mathrm{C} 3-\mathrm{H} 3 \cdots \mathrm{O}^{\mathrm{i}}$ & 0.93 & 2.44 & $3.268(3)$ & 148 \\
$\mathrm{C} 5-\mathrm{H} 5 \cdots 3^{\mathrm{i}}$ & 0.93 & 2.54 & $3.337(3)$ & 144 \\
\hline
\end{tabular}

Symmetry code: (i) $-x+1,-y,-z$.

Data collection: SMART (Bruker, 1998); cell refinement: SAINT (Bruker, 1998); data reduction: $S A I N T$; $\operatorname{program}(\mathrm{s})$ used to solve structure: SHELXS97 (Sheldrick, 2008); program(s) used to refine structure: SHELXL97 (Sheldrick, 2008); molecular graphics: ORTEP-3 for Window (Farrugia, 2012); software used to prepare material for publication: PLATON (Spek, 2009).

\title{
Acknowledgements
}

The authors thank Professor T. N. Guru Row, Indian Institute of Science, Bangalore, for scientific discussions and the data collection.

Supporting information for this paper is available from the IUCr electronic archives (Reference: LH5773).

\section{References}

Ballazhi, L., Popovski, E., Jashari, A., Imeri, F., Ibrahimi, I., Mikhova, B. \& Mladenovska, K. (2015). Acta Pharm. 65, 53-63.

Bruker (1998). SMART and SAINT. Bruker AXS Inc, Madison, Wisconsin, USA.

Chopra, D., Venugopala, K. N. \& Rao, G. K. (2007a). Acta Cryst. E63, o4872.

Chopra, D., Venugopala, K. N., Rao, G. K. \& Guru Row, T. N. (2007b). Acta Cryst. E63, o2826.

Chopra, D., Venugopal, K. N., Jayashree, B. S. \& Row, T. N. G. (2006). Acta Cryst. E62, o2310-02312.

Eid, A. I., Ragab, F. A., El-Ansary, S. L., El-Gazayerly, S. M. \& Mourad, F. E. (1994). Arch. Pharm. Pharm. Med. Chem. 327, 211-213.

Farrugia, L. J. (2012). J. Appl. Cryst. 45, 849-854.

Hooper, D. C., Wolfson, J. S., McHugh, G. L., Winters, M. B. \& Swartz, M. N. (1982). Antimicrob. Agents Chemother. 22, 662-671.

Hsieh, C. (2015). Int. J. Oncol. 46, 2, 555-562.

Kale, M. \& Patwardhan, K. (2014). Curr. Pharm. Res.4, 1150-1158. 


\section{data reports}

Khalfan, H., Abuknesha, R., Rond-Weaver, M., Price, R. G. \& Robinson, R. (1987). Chem. Abstr. 106, 63932.

Lanoot, B., Vancanneyt, M., Cleenwerck, I., Wang, L., Li, W., Liu, Z. \& Swings, J. (2002). Int. J. Syst. Evol. Microbiol. 52, 823-829.

Morris, A. \& Russell, A. D. (1971). Prog. Med. Chem. 8, 39-59.

Munshi, P. \& Guru Row, T. N. (2006). Cryst. Growth Des. 6, 708-718.
Munshi, P., Venugopala, K. N., Jayashree, B. S. \& Guru Row, T. N. (2004). Cryst. Growth Des. 4, 1105-1107.

Sheldrick, G. M. (1996). SADABS. University of Göttingen, Germany.

Sheldrick, G. M. (2008). Acta Cryst. A64, 112-122.

Spek, A. L. (2009). Acta Cryst. D65, 148-155.

Wang, R. (2015). CN Patent CN 104557831 A. 


\title{
supporting information
}

Acta Cryst. (2015). E71, o615-o616 [https://doi.org/10.1107/S2056989015012955]

\section{Crystal structure of 3-bromoacetyl-6-chloro-2H-1-benzopyran-2-one}

\author{
Ramanaiah Chennuru, Balaji Maddimsetti, Suman Gundlapalli, R. Ravi Chandra Babu and \\ Sudarshan Mahapatra
}

\section{S1. Structural commentary}

Coumarins have wide application in the pharmaceutical industry for their antiviral activity (Kale et al., 2014) and antimicrobial activity (Eid et al., 1994). Recently antibacterial activity of the coumarin derivative chloro-chromen-2-one was studied by Lulzime et al., 2015. The coumarin family can also inhibit breast cancer-mediated osteoclastogenesis and this was recently studied (Hsieh et al., 2015; Ballazhi et al., 2015). Further applications of coumarin derivatives for fever, inflammation and pain has been evaluated (Wang et al., 2015). The well known antibiotic Novobiocin (Lanoot et al., 2002; Morris et al., 1971) belongs to coumarin family. The title compound belongs to the 3 -acetyl coumarin family. This coumarin family has potential application in the pharmaceutical field, dye industry and developing LASER dyes (Hooper et al., 1982; Khalfan et al., 1987). The crystal structure of the title coumarin derivative is reported herein.

There are two polymorphic forms of 3-acetyl coumarin reported (Munshi et al., 2004; Munshi et al., 2006). In both cases the structure directing interactions are weak $\mathrm{C}-\mathrm{H} \cdots \mathrm{O}$ hydrogen bonds. In one form (Munshi et al., 2004), a sheetlike structure is formed with two independent molecules in the asymmetric unit and in other form (Munshi et al., 2006) the supramolecular assembly is formed via inter-penetrating sheets with one molecule in the asymmetric unit and contains inversion dimer units connected through weak $\mathrm{C}-\mathrm{H} \cdots \mathrm{O}$ interactions. With the substitution of bromine and chlorine (Chopra et al., 2006;2007a,b) in 3-acetyl coumarin there is no significant differnce in the packing and type of weak interactions. In the crystal of the title compound, pairs of bifurcated $-(\mathrm{C}-\mathrm{H})_{2} \cdots \mathrm{O}$ hydrogen bonds form inversion dimers. The molecular structure of the title compound is shown in Fig. 1.

\section{S2. Synthesis and crystallization}

Synthesis of 3-Bromoacetyl-6-chloro-2H-1-benzopyran-2-one : To a solution of 3-acetyl-6-chloro-2H-1-benzopyran-2one $(222 \mathrm{mg}, 1 \mathrm{mmol})$ in alcohol free chloroform $(5 \mathrm{ml})$, bromine $(173.8 \mathrm{mg}, 1.1 \mathrm{mmol})$ in chloroform $(2 \mathrm{ml})$ was added with intermittent shaking and warming. The mixture was heated for fifteen minutes on a water bath, cooled and filtered. The solid was washed with ether and crystallized from glacial acetic acid to yield 3-bromoacetyl-6-chloro-2H-1-benzopyran-2-one. Needle shape crystals were obtained by dissolving the title compound in glacial acetic acid and warming for a few minutes in a $10 \mathrm{ml}$ beaker. The beaker was covered with paraffin film with few holes in it and left till crystals appeared.

\section{S3. Refinement}

All $\mathrm{H}$ atoms were positioned geometrically and refined using a riding-model approximation with $\mathrm{C}-\mathrm{H}=0.93$ or $0.97 \AA$ and $\mathrm{U}_{\text {iso }}(\mathrm{H})=1.2 \mathrm{U}_{\mathrm{eq}}(\mathrm{C})$. 


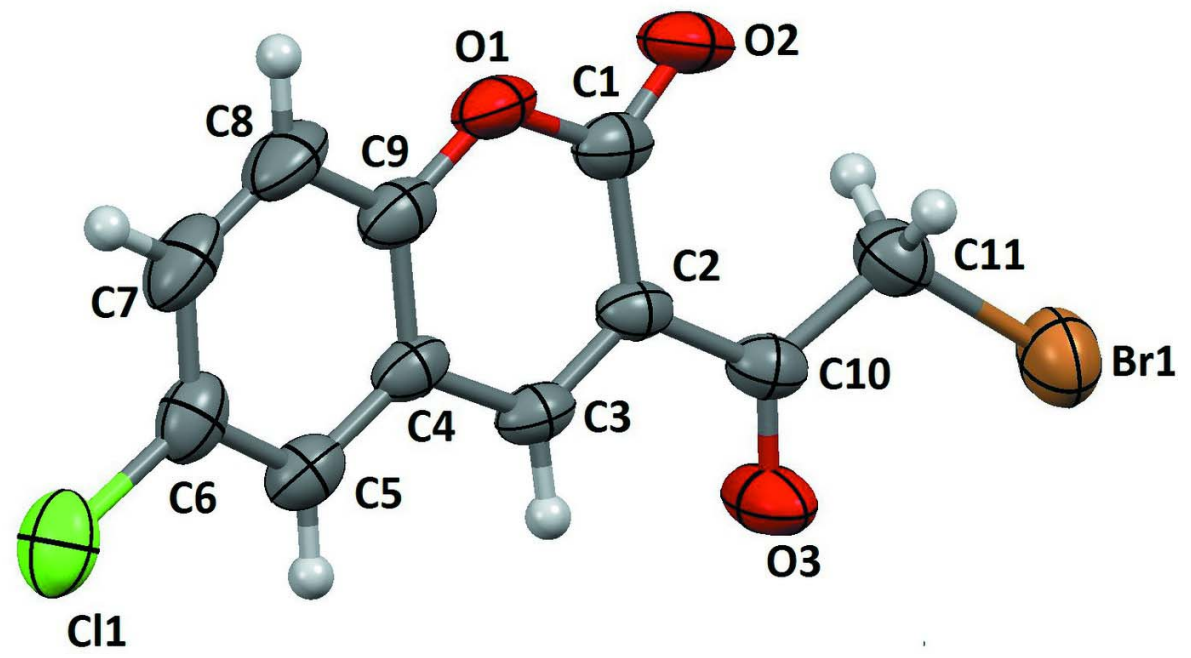

Figure 1

The molecular structure of the title compound with displacement ellipsoids for non- $\mathrm{H}$ atoms drawn at the $50 \%$ probability level.<smiles>CC(=O)c1cc2cc(Cl)ccc2oc1=O</smiles><smiles>O=C(CBr)c1cc2cc(Cl)ccc2oc1=O</smiles>

Figure 2

The reaction scheme.

\section{3-Bromoacetyl-6-chloro-2H-1-benzopyran-2-one}

\section{Crystal data}

$\mathrm{C}_{11} \mathrm{H}_{6} \mathrm{BrClO}_{3}$

$M_{r}=301.51$

Monoclinic, $P 2_{1} / c$

Hall symbol: -P $2 \mathrm{ybc}$

$a=12.5770$ (2) $\AA$

$b=5.7977$ (1) $\AA$

$c=14.8390(3) \AA$

$\beta=94.679(2)^{\circ}$

$V=1078.42(3) \AA^{3}$

$Z=4$

\section{Data collection}

Bruker SMART CCD area-detector diffractometer

Radiation source: fine-focus sealed tube

Graphite monochromator

$\varphi$ and $\omega$ scans

Absorption correction: multi-scan

(SADABS; Sheldrick, 1996)

$T_{\min }=0.295, T_{\max }=0.712$
$F(000)=592$

$D_{\mathrm{x}}=1.857 \mathrm{Mg} \mathrm{m}^{-3}$

Mo $K \alpha$ radiation, $\lambda=0.71073 \AA$

Cell parameters from 2113 reflections

$\theta=3.1-26.0^{\circ}$

$\mu=4.05 \mathrm{~mm}^{-1}$

$T=293 \mathrm{~K}$

Needle, yellow

$0.40 \times 0.10 \times 0.09 \mathrm{~mm}$

20627 measured reflections

2113 independent reflections

1532 reflections with $I>2 \sigma(I)$

$R_{\text {int }}=0.031$

$\theta_{\text {max }}=26.0^{\circ}, \theta_{\text {min }}=3.1^{\circ}$

$h=-15 \rightarrow 15$

$k=-7 \rightarrow 7$

$l=-18 \rightarrow 18$ 


\section{Refinement}

Refinement on $F^{2}$

Least-squares matrix: full

$R\left[F^{2}>2 \sigma\left(F^{2}\right)\right]=0.030$

$w R\left(F^{2}\right)=0.076$

$S=0.95$

2113 reflections

145 parameters

0 restraints

Primary atom site location: structure-invariant direct methods
Secondary atom site location: difference Fourier map

Hydrogen site location: inferred from neighbouring sites

$\mathrm{H}$-atom parameters constrained

$w=1 /\left[\sigma^{2}\left(F_{\mathrm{o}}^{2}\right)+(0.0424 P)^{2}+0.3438 P\right]$ where $P=\left(F_{\mathrm{o}}{ }^{2}+2 F_{\mathrm{c}}{ }^{2}\right) / 3$

$(\Delta / \sigma)_{\max }=0.001$

$\Delta \rho_{\max }=0.43 \mathrm{e} \AA^{-3}$

$\Delta \rho_{\min }=-0.60$ e $\AA^{-3}$

Special details

Geometry. Bond distances, angles etc. have been calculated using the rounded fractional coordinates. All su's are estimated from the variances of the (full) variance-covariance matrix. The cell e.s.d.'s are taken into account in the estimation of distances, angles and torsion angles

Refinement. Refinement of $F^{2}$ against ALL reflections. The weighted $R$-factor $w R$ and goodness of fit $S$ are based on $F^{2}$, conventional $R$-factors $R$ are based on $F$, with $F$ set to zero for negative $F^{2}$. The threshold expression of $F^{2}>\sigma\left(F^{2}\right)$ is used only for calculating $R$-factors (gt) etc. and is not relevant to the choice of reflections for refinement. $R$-factors based on $F^{2}$ are statistically about twice as large as those based on $F$, and $R$ - factors based on ALL data will be even larger.

Fractional atomic coordinates and isotropic or equivalent isotropic displacement parameters $\left(\AA^{2}\right)$

\begin{tabular}{lllll}
\hline & $x$ & $y$ & $z$ & $U_{\text {iso }} / U_{\text {eq }}$ \\
\hline Br1 & $0.82908(2)$ & $0.43513(6)$ & $0.08551(2)$ & $0.0724(1)$ \\
C11 & $0.03785(6)$ & $-0.08546(14)$ & $0.11305(5)$ & $0.0667(3)$ \\
O1 & $0.36571(16)$ & $0.6170(3)$ & $0.19323(11)$ & $0.0522(6)$ \\
O2 & $0.52243(17)$ & $0.7733(3)$ & $0.18198(12)$ & $0.0592(7)$ \\
O3 & $0.62089(15)$ & $0.2132(3)$ & $0.03489(14)$ & $0.0665(7)$ \\
C1 & $0.4668(2)$ & $0.6090(4)$ & $0.16473(15)$ & $0.0450(9)$ \\
C2 & $0.4945(2)$ & $0.4025(4)$ & $0.11494(14)$ & $0.0380(8)$ \\
C3 & $0.4205(2)$ & $0.2395(4)$ & $0.09438(15)$ & $0.0396(8)$ \\
C4 & $0.3152(2)$ & $0.2556(4)$ & $0.12289(15)$ & $0.0403(8)$ \\
C5 & $0.2359(2)$ & $0.0892(4)$ & $0.10347(16)$ & $0.0453(8)$ \\
C6 & $0.1383(2)$ & $0.1173(5)$ & $0.13694(17)$ & $0.0507(9)$ \\
C7 & $0.1178(3)$ & $0.3055(6)$ & $0.19074(19)$ & $0.0624(10)$ \\
C8 & $0.1941(3)$ & $0.4691(6)$ & $0.21009(19)$ & $0.0616(11)$ \\
C9 & $0.2920(2)$ & $0.4454(4)$ & $0.17544(16)$ & $0.0476(8)$ \\
C10 & $0.6045(2)$ & $0.3676(4)$ & $0.08674(15)$ & $0.0422(8)$ \\
C11 & $0.6926(2)$ & $0.5239(5)$ & $0.12365(18)$ & $0.0546(9)$ \\
H3 & 0.43848 & 0.11242 & 0.06054 & $0.0475^{*}$ \\
H5 & 0.24924 & -0.03874 & 0.06832 & $0.0543^{*}$ \\
H7 & 0.05146 & 0.32003 & 0.21373 & $0.0747^{*}$ \\
H8 & 0.18033 & 0.59506 & 0.24616 & $0.0741^{*}$ \\
H11A & 0.67683 & 0.68051 & 0.10379 & $0.0655^{*}$ \\
H11B & 0.69582 & 0.52191 & 0.18918 & $0.0655^{*}$ \\
& & & &
\end{tabular}


Atomic displacement parameters $\left(\AA^{2}\right)$

\begin{tabular}{lllllll}
\hline & $U^{11}$ & $U^{22}$ & $U^{33}$ & $U^{12}$ & $U^{13}$ & $U^{23}$ \\
\hline Br1 & $0.0534(2)$ & $0.0854(3)$ & $0.0780(2)$ & $-0.0081(2)$ & $0.0030(2)$ & $-0.0066(2)$ \\
C11 & $0.0485(4)$ & $0.0861(6)$ & $0.0667(4)$ & $0.0015(4)$ & $0.0129(3)$ & $0.0141(4)$ \\
O1 & $0.0676(13)$ & $0.0426(10)$ & $0.0454(10)$ & $0.0208(9)$ & $-0.0008(9)$ & $-0.0103(8)$ \\
O2 & $0.0855(15)$ & $0.0367(10)$ & $0.0539(11)$ & $0.0024(10)$ & $-0.0027(10)$ & $-0.0138(9)$ \\
O3 & $0.0569(12)$ & $0.0673(13)$ & $0.0782(13)$ & $-0.0123(10)$ & $0.0231(10)$ & $-0.0361(12)$ \\
C1 & $0.0644(18)$ & $0.0372(15)$ & $0.0318(12)$ & $0.0127(13)$ & $-0.0050(12)$ & $0.0010(11)$ \\
C2 & $0.0543(15)$ & $0.0308(13)$ & $0.0285(11)$ & $0.0060(11)$ & $0.0008(10)$ & $-0.0006(10)$ \\
C3 & $0.0543(15)$ & $0.0338(13)$ & $0.0313(12)$ & $0.0110(12)$ & $0.0075(11)$ & $-0.0007(10)$ \\
C4 & $0.0516(15)$ & $0.0379(14)$ & $0.0315(12)$ & $0.0154(12)$ & $0.0049(10)$ & $0.0030(10)$ \\
C5 & $0.0509(15)$ & $0.0454(15)$ & $0.0405(13)$ & $0.0126(13)$ & $0.0096(11)$ & $0.0052(11)$ \\
C6 & $0.0472(16)$ & $0.0639(18)$ & $0.0411(14)$ & $0.0130(13)$ & $0.0050(12)$ & $0.0120(13)$ \\
C7 & $0.0523(18)$ & $0.083(2)$ & $0.0535(16)$ & $0.0289(17)$ & $0.0142(14)$ & $0.0042(16)$ \\
C8 & $0.065(2)$ & $0.069(2)$ & $0.0515(16)$ & $0.0325(17)$ & $0.0086(14)$ & $-0.0107(14)$ \\
C9 & $0.0573(16)$ & $0.0468(15)$ & $0.0383(12)$ & $0.0195(14)$ & $0.0013(11)$ & $0.0005(12)$ \\
C10 & $0.0558(16)$ & $0.0361(13)$ & $0.0348(12)$ & $-0.0014(11)$ & $0.0036(11)$ & $-0.0017(11)$ \\
C11 & $0.0595(18)$ & $0.0532(16)$ & $0.0497(15)$ & $-0.0032(13)$ & $-0.0034(13)$ & $-0.0071(13)$ \\
& & & & & & \\
\hline
\end{tabular}

Geometric parameters $\left(\AA,{ }^{\circ}\right)$

\begin{tabular}{|c|c|c|c|}
\hline $\mathrm{Br} 1-\mathrm{C} 11$ & $1.921(3)$ & $\mathrm{C} 5-\mathrm{C} 6$ & $1.371(4)$ \\
\hline $\mathrm{C} 11-\mathrm{C} 6$ & $1.741(3)$ & $\mathrm{C} 6-\mathrm{C} 7$ & $1.389(4)$ \\
\hline $\mathrm{O} 1-\mathrm{C} 1$ & $1.373(3)$ & $\mathrm{C} 7-\mathrm{C} 8$ & $1.363(5)$ \\
\hline $\mathrm{O} 1-\mathrm{C} 9$ & $1.371(3)$ & $\mathrm{C} 8-\mathrm{C} 9$ & $1.379(4)$ \\
\hline $\mathrm{O} 2-\mathrm{C} 1$ & $1.197(3)$ & $\mathrm{C} 10-\mathrm{C} 11$ & $1.500(4)$ \\
\hline $\mathrm{O} 3-\mathrm{C} 10$ & $1.209(3)$ & $\mathrm{C} 3-\mathrm{H} 3$ & 0.9300 \\
\hline $\mathrm{C} 1-\mathrm{C} 2$ & $1.464(3)$ & $\mathrm{C} 5-\mathrm{H} 5$ & 0.9300 \\
\hline $\mathrm{C} 2-\mathrm{C} 3$ & $1.344(3)$ & $\mathrm{C} 7-\mathrm{H} 7$ & 0.9300 \\
\hline $\mathrm{C} 2-\mathrm{C} 10$ & $1.492(4)$ & $\mathrm{C} 8-\mathrm{H} 8$ & 0.9300 \\
\hline $\mathrm{C} 3-\mathrm{C} 4$ & $1.426(4)$ & C11-H11A & 0.9700 \\
\hline $\mathrm{C} 4-\mathrm{C} 5$ & $1.401(3)$ & C11-H11B & 0.9700 \\
\hline $\mathrm{C} 4-\mathrm{C} 9$ & $1.393(3)$ & & \\
\hline $\mathrm{Br} 1 \cdots \mathrm{O} 3$ & 2.9589 (19) & $\mathrm{C} 2 \cdots \mathrm{C} 10^{\text {viii }}$ & $3.417(3)$ \\
\hline $\mathrm{Br} 1 \cdots \mathrm{H}^{\mathrm{i}}$ & 3.1900 & $\mathrm{C} 2 \cdots \mathrm{O} 3^{\text {viii }}$ & $3.389(3)$ \\
\hline $\mathrm{Cl1} \cdots \mathrm{C}^{\mathrm{ii}}$ & $3.485(4)$ & $\mathrm{C} 3 \cdots \mathrm{O} 2^{\mathrm{i}}$ & $3.343(3)$ \\
\hline $\mathrm{C} 11 \cdots \mathrm{C} 11^{\mathrm{iii}}$ & $3.5530(11)$ & $\mathrm{C} 3 \cdots \mathrm{O} 2^{\mathrm{ii}}$ & $3.220(3)$ \\
\hline $\mathrm{Cl} 1 \cdots \mathrm{H} 7^{\text {iv }}$ & 2.9400 & $\mathrm{C} 3 \cdots \mathrm{C} 10^{\text {viii }}$ & $3.518(3)$ \\
\hline $\mathrm{O} 1 \cdots \mathrm{C}^{\mathrm{v}}$ & $3.403(3)$ & $\mathrm{C} 3 \cdots \mathrm{O} 3^{\mathrm{vii}}$ & $3.268(3)$ \\
\hline $\mathrm{O} 1 \cdots \mathrm{O} 2^{\mathrm{i}}$ & $2.992(3)$ & $\mathrm{C} 4 \cdots \mathrm{O} 2^{\mathrm{i}}$ & $3.406(3)$ \\
\hline $\mathrm{O} 2 \cdots \mathrm{C} 3^{\mathrm{v}}$ & $3.220(3)$ & $\mathrm{C} 5 \cdots \mathrm{O} 3^{\text {vii }}$ & $3.337(3)$ \\
\hline $\mathrm{O} 2 \cdots \mathrm{C} 11$ & $2.779(3)$ & $\mathrm{C} 5 \cdots \mathrm{O} 1^{\mathrm{ii}}$ & $3.403(3)$ \\
\hline $\mathrm{O} 2 \cdots \mathrm{C} 4^{\mathrm{vi}}$ & $3.406(3)$ & $\mathrm{C} 8 \cdots \mathrm{Cl}^{\mathrm{v}}$ & $3.485(4)$ \\
\hline $\mathrm{O} 2 \cdots \mathrm{C} 9^{\text {vi }}$ & $3.180(3)$ & $\mathrm{C} 9 \cdots \mathrm{O} 2^{\mathrm{i}}$ & $3.180(3)$ \\
\hline $\mathrm{O} 2 \cdots \mathrm{C} 2^{\mathrm{vi}}$ & $3.129(3)$ & $\mathrm{C} 10 \cdots \mathrm{C} 2^{\text {viii }}$ & $3.417(3)$ \\
\hline $\mathrm{O} 2 \cdots \mathrm{O} 1^{\mathrm{vi}}$ & $2.992(3)$ & $\mathrm{C} 10 \cdots \mathrm{C} 3^{\text {viii }}$ & $3.518(3)$ \\
\hline
\end{tabular}




\begin{tabular}{|c|c|c|c|}
\hline $\mathrm{O} 2 \cdots \mathrm{C} 1^{\mathrm{vi}}$ & $2.988(3)$ & $\mathrm{C} 11 \cdots \mathrm{O} 2$ & $2.779(3)$ \\
\hline $\mathrm{O} 2 \cdots \mathrm{C} 3^{\mathrm{vi}}$ & $3.343(3)$ & $\mathrm{C} 1 \cdots \mathrm{H} 11 \mathrm{~B}$ & 2.9200 \\
\hline $\mathrm{O} 3 \cdots \mathrm{Br} 1$ & $2.9589(19)$ & $\mathrm{C} 1 \cdots \mathrm{H} 11 \mathrm{~A}$ & 2.8900 \\
\hline $\mathrm{O} 3 \cdots \mathrm{C} 5^{\text {vii }}$ & $3.337(3)$ & $\mathrm{H} 3 \cdots \mathrm{O} 2^{\mathrm{ii}}$ & 2.8100 \\
\hline $\mathrm{O} 3 \cdots \mathrm{C} 1^{\text {viii }}$ & $3.244(3)$ & $\mathrm{H} 3 \cdots \mathrm{O} 3$ & 2.4300 \\
\hline $\mathrm{O} 3 \cdots \mathrm{C} 2^{\text {viii }}$ & $3.389(3)$ & H3 $\cdots$ H5 & 2.5500 \\
\hline $\mathrm{O} 3 \cdots \mathrm{C} 3^{\mathrm{vii}}$ & $3.268(3)$ & $\mathrm{H} 3 \cdots \mathrm{O} 3^{\text {vii }}$ & 2.4400 \\
\hline $\mathrm{O} 2 \cdots \mathrm{H} 11 \mathrm{~A}$ & 2.4000 & 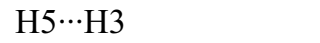 & 2.5500 \\
\hline $\mathrm{O} 2 \cdots \mathrm{H} 11 \mathrm{~B}$ & 2.6200 & $\mathrm{H} 5 \cdots \mathrm{O} 3^{\text {vii }}$ & 2.5400 \\
\hline $\mathrm{O} 2 \cdots \mathrm{H} 3^{v}$ & 2.8100 & $\mathrm{H} 7 \cdots \mathrm{Cl} 1^{\text {ix }}$ & 2.9400 \\
\hline $\mathrm{O} 3 \cdots \mathrm{H} 3$ & 2.4300 & $\mathrm{H} 8 \cdots \mathrm{Br}^{\mathrm{vi}}$ & 3.1900 \\
\hline $\mathrm{O} 3 \cdots{ }^{*} 3^{\text {vii }}$ & 2.4400 & $\mathrm{H} 11 \mathrm{~A} \cdots \mathrm{O} 2$ & 2.4000 \\
\hline $\mathrm{O} 3 \cdots \mathrm{H}^{\mathrm{vii}}$ & 2.5400 & $\mathrm{H} 11 \mathrm{~A} \cdots \mathrm{C} 1$ & 2.8900 \\
\hline $\mathrm{C} 1 \cdots \mathrm{O} 3^{\text {viii }}$ & $3.244(3)$ & $\mathrm{H} 11 \mathrm{~B} \cdots \mathrm{O} 2$ & 2.6200 \\
\hline $\mathrm{C} 1 \cdots \mathrm{O} 2^{\mathrm{i}}$ & $2.988(3)$ & $\mathrm{H} 11 \mathrm{~B} \cdots \mathrm{C} 1$ & 2.9200 \\
\hline $\mathrm{C} 2 \cdots \mathrm{O} 2^{\mathrm{i}}$ & $3.129(3)$ & & \\
\hline $\mathrm{C} 1-\mathrm{O} 1-\mathrm{C} 9$ & $123.02(19)$ & $\mathrm{C} 4-\mathrm{C} 9-\mathrm{C} 8$ & $121.4(2)$ \\
\hline $\mathrm{O} 1-\mathrm{C} 1-\mathrm{O} 2$ & $116.6(2)$ & $\mathrm{O} 3-\mathrm{C} 10-\mathrm{C} 2$ & $119.3(2)$ \\
\hline $\mathrm{O} 1-\mathrm{C} 1-\mathrm{C} 2$ & $116.6(2)$ & $\mathrm{O} 3-\mathrm{C} 10-\mathrm{C} 11$ & $121.4(2)$ \\
\hline $\mathrm{O} 2-\mathrm{C} 1-\mathrm{C} 2$ & $126.9(2)$ & $\mathrm{C} 2-\mathrm{C} 10-\mathrm{C} 11$ & $119.4(2)$ \\
\hline $\mathrm{C} 1-\mathrm{C} 2-\mathrm{C} 3$ & $120.1(2)$ & $\mathrm{Br} 1-\mathrm{C} 11-\mathrm{C} 10$ & $112.44(18)$ \\
\hline $\mathrm{C} 1-\mathrm{C} 2-\mathrm{C} 10$ & $121.1(2)$ & $\mathrm{C} 2-\mathrm{C} 3-\mathrm{H} 3$ & 119.00 \\
\hline $\mathrm{C} 3-\mathrm{C} 2-\mathrm{C} 10$ & $118.8(2)$ & $\mathrm{C} 4-\mathrm{C} 3-\mathrm{H} 3$ & 119.00 \\
\hline $\mathrm{C} 2-\mathrm{C} 3-\mathrm{C} 4$ & $122.0(2)$ & $\mathrm{C} 4-\mathrm{C} 5-\mathrm{H} 5$ & 120.00 \\
\hline $\mathrm{C} 3-\mathrm{C} 4-\mathrm{C} 5$ & $123.8(2)$ & $\mathrm{C} 6-\mathrm{C} 5-\mathrm{H} 5$ & 120.00 \\
\hline $\mathrm{C} 3-\mathrm{C} 4-\mathrm{C} 9$ & $117.4(2)$ & $\mathrm{C} 6-\mathrm{C} 7-\mathrm{H} 7$ & 120.00 \\
\hline $\mathrm{C} 5-\mathrm{C} 4-\mathrm{C} 9$ & $118.8(2)$ & $\mathrm{C} 8-\mathrm{C} 7-\mathrm{H} 7$ & 120.00 \\
\hline $\mathrm{C} 4-\mathrm{C} 5-\mathrm{C} 6$ & $119.2(2)$ & $\mathrm{C} 7-\mathrm{C} 8-\mathrm{H} 8$ & 120.00 \\
\hline $\mathrm{C} 11-\mathrm{C} 6-\mathrm{C} 5$ & $120.2(2)$ & $\mathrm{C} 9-\mathrm{C} 8-\mathrm{H} 8$ & 120.00 \\
\hline $\mathrm{C} 11-\mathrm{C} 6-\mathrm{C} 7$ & $118.8(2)$ & $\mathrm{Br} 1-\mathrm{C} 11-\mathrm{H} 11 \mathrm{~A}$ & 109.00 \\
\hline $\mathrm{C} 5-\mathrm{C} 6-\mathrm{C} 7$ & $121.0(3)$ & $\mathrm{Br} 1-\mathrm{C} 11-\mathrm{H} 11 \mathrm{~B}$ & 109.00 \\
\hline $\mathrm{C} 6-\mathrm{C} 7-\mathrm{C} 8$ & $120.5(3)$ & $\mathrm{C} 10-\mathrm{C} 11-\mathrm{H} 11 \mathrm{~A}$ & 109.00 \\
\hline $\mathrm{C} 7-\mathrm{C} 8-\mathrm{C} 9$ & $119.1(3)$ & $\mathrm{C} 10-\mathrm{C} 11-\mathrm{H} 11 \mathrm{~B}$ & 109.00 \\
\hline $\mathrm{O} 1-\mathrm{C} 9-\mathrm{C} 4$ & $120.8(2)$ & $\mathrm{H} 11 \mathrm{~A}-\mathrm{C} 11-\mathrm{H} 11 \mathrm{~B}$ & 108.00 \\
\hline $\mathrm{O} 1-\mathrm{C} 9-\mathrm{C} 8$ & $117.8(2)$ & & \\
\hline $\mathrm{C} 9-\mathrm{O} 1-\mathrm{C} 1-\mathrm{O} 2$ & $177.6(2)$ & $\mathrm{C} 3-\mathrm{C} 4-\mathrm{C} 5-\mathrm{C} 6$ & $177.6(2)$ \\
\hline $\mathrm{C} 9-\mathrm{O} 1-\mathrm{C} 1-\mathrm{C} 2$ & $-1.3(3)$ & $\mathrm{C} 9-\mathrm{C} 4-\mathrm{C} 5-\mathrm{C} 6$ & $-0.3(3)$ \\
\hline $\mathrm{C} 1-\mathrm{O} 1-\mathrm{C} 9-\mathrm{C} 4$ & $-3.0(3)$ & $\mathrm{C} 3-\mathrm{C} 4-\mathrm{C} 9-\mathrm{O} 1$ & $4.5(3)$ \\
\hline $\mathrm{C} 1-\mathrm{O} 1-\mathrm{C} 9-\mathrm{C} 8$ & $177.9(2)$ & $\mathrm{C} 3-\mathrm{C} 4-\mathrm{C} 9-\mathrm{C} 8$ & $-176.5(2)$ \\
\hline $\mathrm{O} 1-\mathrm{C} 1-\mathrm{C} 2-\mathrm{C} 3$ & $4.1(3)$ & $\mathrm{C} 5-\mathrm{C} 4-\mathrm{C} 9-\mathrm{O} 1$ & $-177.5(2)$ \\
\hline $\mathrm{O} 1-\mathrm{C} 1-\mathrm{C} 2-\mathrm{C} 10$ & $-175.44(19)$ & $\mathrm{C} 5-\mathrm{C} 4-\mathrm{C} 9-\mathrm{C} 8$ & $1.6(4)$ \\
\hline $\mathrm{O} 2-\mathrm{C} 1-\mathrm{C} 2-\mathrm{C} 3$ & $-174.6(2)$ & $\mathrm{C} 4-\mathrm{C} 5-\mathrm{C} 6-\mathrm{Cl} 1$ & $179.43(19)$ \\
\hline $\mathrm{O} 2-\mathrm{C} 1-\mathrm{C} 2-\mathrm{C} 10$ & $5.9(4)$ & $\mathrm{C} 4-\mathrm{C} 5-\mathrm{C} 6-\mathrm{C} 7$ & $-1.1(4)$ \\
\hline $\mathrm{C} 1-\mathrm{C} 2-\mathrm{C} 3-\mathrm{C} 4$ & $-2.7(3)$ & $\mathrm{C} 11-\mathrm{C} 6-\mathrm{C} 7-\mathrm{C} 8$ & $-179.3(2)$ \\
\hline $\mathrm{C} 10-\mathrm{C} 2-\mathrm{C} 3-\mathrm{C} 4$ & $176.9(2)$ & $\mathrm{C} 5-\mathrm{C} 6-\mathrm{C} 7-\mathrm{C} 8$ & $1.2(4)$ \\
\hline $\mathrm{C} 1-\mathrm{C} 2-\mathrm{C} 10-\mathrm{O} 3$ & $-169.4(2)$ & $\mathrm{C} 6-\mathrm{C} 7-\mathrm{C} 8-\mathrm{C} 9$ & $0.1(4)$ \\
\hline
\end{tabular}




$\begin{array}{llll}\mathrm{C} 1-\mathrm{C} 2-\mathrm{C} 10-\mathrm{C} 11 & 10.9(3) & \mathrm{C} 7-\mathrm{C} 8-\mathrm{C} 9-\mathrm{O} 1 & 177.6(3) \\ \mathrm{C} 3-\mathrm{C} 2-\mathrm{C} 10-\mathrm{O} 3 & 11.0(3) & \mathrm{C} 7-\mathrm{C} 8-\mathrm{C} 9-\mathrm{C} 4 & -1.4(4) \\ \mathrm{C} 3-\mathrm{C} 2-\mathrm{C} 10-\mathrm{C} 11 & -168.7(2) & \mathrm{O} 3-\mathrm{C} 10-\mathrm{C} 11-\mathrm{Br} 1 & -3.9(3) \\ \mathrm{C} 2-\mathrm{C} 3-\mathrm{C} 4-\mathrm{C} 5 & -179.6(2) & \mathrm{C} 2-\mathrm{C} 10-\mathrm{C} 11-\mathrm{Br} 1 & 175.83(17) \\ \mathrm{C} 2-\mathrm{C} 3-\mathrm{C} 4-\mathrm{C} 9 & -1.6(3) & & \end{array}$

Symmetry codes: (i) $-x+1, y-1 / 2,-z+1 / 2$; (ii) $x, y-1, z$; (iii) $-x,-y,-z$; (iv) $-x, y-1 / 2,-z+1 / 2$; (v) $x, y+1, z$; (vi) $-x+1, y+1 / 2,-z+1 / 2$; (vii) $-x+1,-y,-z$; (viii) $-x+1,-y+1,-z$; (ix) $-x, y+1 / 2,-z+1 / 2$.

Hydrogen-bond geometry $\left(\AA,{ }^{\circ}\right)$

\begin{tabular}{lllll}
\hline$D-\mathrm{H} \cdots A$ & $D-\mathrm{H}$ & $\mathrm{H} \cdots A$ & $D \cdots A$ & $D-\mathrm{H} \cdots A$ \\
\hline $\mathrm{C} 3-\mathrm{H} 3 \cdots \mathrm{O} 3^{\text {vii }}$ & 0.9300 & 2.4400 & $3.268(3)$ & 148.00 \\
$\mathrm{C} 5-\mathrm{H} 5 \cdots 3^{\text {vii }}$ & 0.9300 & 2.5400 & $3.337(3)$ & 144.00 \\
\hline
\end{tabular}

Symmetry code: (vii) $-x+1,-y,-z$. 